\title{
Sorting task as a rapid tool to explore sensory shelf-life in food products: a case study using smoothies of exotic and red fruit flavours
}

\author{
E. Campo ${ }^{1}$, E. Arias ${ }^{2}$, M.E. Venturini ${ }^{1}$, S. Remón ${ }^{1}$ and R. Oriaa ${ }^{1, a}$
}

1Plant Food Research Group, Instituto Agroalimentario de Aragón - IA2, Universidad de Zaragoza-CITA, Miguel Servet, 177, 50013, Zaragoza, Spain; ${ }^{2}$ PCTAD, Aula Dei Scientific and Technological Park, Avenida Montañana $\mathrm{n}^{\circ}$ 930, 50059, Zaragoza, Spain.

\begin{abstract}
Storage life of most food products is limited by changes in their sensory characteristics. The present work shows how a sorting strategy can be a fast and reliable tool to quickly explore sensory deterioration in perishable products, using fruit smoothies under development as a case study. Smoothies (exotic and red fruit flavours) at three storage times were sorted by 30 consumers according to their global sensory similarities to check if replicates at to were globally perceived as similar among them, and different from samples with longer storage time. Samples were also submitted to physicochemical, nutritional and microbiological analysis. A panel of 12 trained panellists described both prototype flavours at to, and six commercial smoothies to characterize freshly bottled products. If smoothies fulfil a quality standard when bottled - susceptible of being spoiled during storage - the sorting procedure can provide a fast and reliable estimation of attributes inducing a loss of sensory quality. Without any list of descriptors, the terms provided by consumers to characterize the sorted smoothies were equivalent to those mostly employed by the trained panel. This approach could be implemented in food industries in order to monitor sensory decay during storage.
\end{abstract}

Keywords: sorting, sensory deterioration, descriptive analysis, smoothies, cold storage

\section{INTRODUCTION}

Smoothies are blended beverages containing fruit, fruit juice, ice, yoghurt and milk that are oriented to ready-to-drink consumption. They have a "healthy" image and are considered to be a high quality and nutritious product with a fresh-like flavour and appearance. The storage life of these products can be short due to their ability to support microbial growth as a result of the minimal level of processing associated with these products. However, even if smoothies achieve a microbial stability, their sensory properties can be a limiting factor during cold storage (Hough and Garitta, 2012) as a result of flavour deterioration.

During the last decade, the industry demands for quick and reliable screening tools to monitor product quality during manufacturing has boosted the development of low cost and less time consuming methods ready to be applied on site (Varela and Ares, 2012). Sorting task, a simple procedure in which participants are asked to examine samples and to group them according to similarity (odour, flavour, colour, etc.), has become a very popular way to obtain fast sensory information about food and non-food products. Despite a few differences, perceptual maps obtained from sorting tasks are globally comparable with those obtained from classical descriptive analysis (Varela and Ares, 2012). A comprehensive review about the advantages and limitations of sorting task applied to sensory research carried out by Chollet et al. (2011) highlighted that this technique could be an interesting tool for quality control purposes in food industries to guarantee quality standards.

The main objective of this research will be therefore to evaluate if the eventual sensory decay of fruit smoothies can be monitored by relatively simple tasks based on a sorting

aE-mail: oria@posta.unizar.es 
strategy carried out by untrained participants (consumers). For this purpose, smoothies elaborated with two main ingredients (exotic fruits (pineapple, mango and passion fruit) or red fruits (strawberry and raspberry) at three storage times, freshly bottled, at 14 days and 28 days were employed. Additionally, the analysis of the microbial counts was performed to check for evolution of these quality parameters during storage time. In parallel, a classical descriptive analysis made by a trained panel will be performed to characterize freshly bottled smoothies (t0), when the product is expected to exhibit its optimum organoleptic quality.

\section{MATERIALS AND METHODS}

\section{Samples}

Two smoothies of exotic fruits (pineapple, mango and passion fruit) and red fruits (strawberry and raspberry) as main flavours were provided by a juice producer from Zaragoza province (North-West of Spain). These products are under development (not yet commercialized) and had received a high hydrostatic pressure (HHP) processing to ensure adequate stability and maximum retention of nutritional and organoleptic properties. Both smoothies were simultaneously supplied by the producer at three different storage times freshly bottled ( $\mathrm{t} 0$ ), and after 14 and 28 days of cold storage at $4^{\circ} \mathrm{C}(\mathrm{t} 14$ and $\mathrm{t} 28$, respectively) - in order to perform the sorting experiment. This type of experimental design is called reversed storage design and has the advantages of overcoming the major drawbacks of basic storage design (Hough and Garitta, 2012). Six commercial smoothies were also included in the study for descriptive analysis purposes. Samples were selected based on market share and flavor types and covered a wide range of the exotic and red fruits-like smoothie drinks commercially available on the Spanish market.

\section{Microbiological counts}

Microbial analysis included the enumeration of mesophilic aerobic counts on day 0 and after 14 and 28 days at $4^{\circ} \mathrm{C}$. Ten mL of each sample was obtained aseptically and homogenized with $90 \mathrm{~mL}$ of sterile distilled peptone water $0.1 \%$ (Merck, Darmstadt, Germany). Further decimal dilutions were made with the same diluent, and duplicates of at least three appropriate dilutions were plated on appropriate media. Each dilution (1 mL) was pourplated on plate count agar (PCA; Merck, Darmstadt, Germany). After incubation at $30^{\circ} \mathrm{C}$ for 72 h, plates with 15-300 colonies were counted. Microbial data were transformed into logarithms of the number of colony-forming units ( $\left.\log \mathrm{CFU} \mathrm{mL} \mathrm{m}^{-1}\right)$. Three separate samples of each type of smoothie were analysed.

\section{Sorting task}

\section{Participants.}

A total of 32 consumers (18 women and 14 men) aged between 18 and 52 years (mean age $=31$ years) participated in the study. They were staff members and students of the Food Science Faculty of the University of Zaragoza (Spain) and were regular consumers of fruit smoothies.

\section{Procedure.}

Each consumer participated in one 45-min session where they evaluated the two sets of samples: exotic fruits and red fruit samples. For each smoothie, consumers were provided with the 6 samples ( 2 replicates of each t0, $\mathrm{t} 14$ and $\mathrm{t} 28$ ) of the set in $15 \mathrm{~mL}$ transparent plastic glasses coded with different three-digit numbers and arranged in random order. They were asked to sort the 6 samples on the basis of similarity pertaining to the global sensations perceived in mouth. Participants were free to make as many groups as they wished with as many smoothies per group. After the completion of the task, the panellists were asked to provide a few words to describe each of the groups they had formed. The panellists were not informed about the nature of the samples. Experiments were conducted in a sensory laboratory with individual testing booths equipped with serving windows under white light. 
Mineral water and unsalted bread crackers were available for assessors to rinse between samples.

\section{Data analysis.}

Encoding free sorting data.

For each subject, results were encoded in an individual similarity matrix (smoothies $\times$ smoothies), in which 1 stands for two smoothies set in the same group and 0 for two smoothies put in different groups. These individual matrices were summed across subjects; the resulting co-occurrence matrix represents the global similarity matrix where larger numbers indicate higher similarity between samples. The assumption underlying this method is that samples grouped together are more similar than samples sorted into different groups.

\section{Multidimensional scaling and cluster analysis.}

The resulting co-occurrence matrix was submitted to an MDS analysis in order to derive a spatial representation of samples. A non-metric scaling algorithm was used (alternating least-square scaling). The quality of fit was measured by the normalized stress value. Coordinates of samples in the retained MDS configuration were submitted to a hierarchical cluster analysis with the Ward criterion. HCA makes it possible to check the spatial arrangement of MDS data and refine the interpretation of the map by identifying the smoothies that belong to a same cluster. All analyses were performed with the software SPSS 15.0 (SPSS Inc., Chicago, IL, USA).

\section{Analysis of vocabulary.}

For each consumer, the terms given to characterize each established group were associated to each smoothie of the group. The underlying assumption within this approach is that all the samples belonging to the same group were described by the terms in the same way. After grouping synonyms, the frequency of quotation of each term was calculated for each sample.

\section{Descriptive analysis}

\section{Panel.}

A group of 12 members, 5 male and 7 females ranging from 24 to 65 years old (average = 33) was selected and trained according to the guidelines set out in ISO 8586-1 (1993) and in ISO NORM 11035 (1994), respectively. The panellists were selected from staff and graduate students of the Food Science Faculty at University of Zaragoza.

\section{Procedure.}

Panel members were trained and a set of descriptors was developed and defined for smoothie samples during training, which included aroma, flavour and textural attributes. Thirteen descriptors were developed and agreed upon by panel consensus. Intensity ratings were scored using a 10 point scale anchored "not perceived" to "strongly perceived". Texture was evaluated during scooping and mastication in mouth. Sensory profiling was carried out in a sensory laboratory with individual testing booths equipped with serving windows. Mineral water and bread crackers were available for assessors. Panellists participated in four $1 \mathrm{~h}$ sessions to evaluate samples in duplicate. They evaluated 8 samples in a randomised order (Macfie et al., 1989).

\section{Data analysis.}

Standardized principal component analysis (PCA) was performed on the mean ratings among the panellists for all attributes and for each smoothie (correlation matrix). Dimensions with an eigenvalue higher than the mean eigenvalue (Kaiser law) were retained. All analyses were carried out with SPAD software (version 5.5, CISIA-CESRESTA, Montreuil, France). 


\section{RESULTS}

\section{Microbial counts}

Samples of HPP processed exotic and red fruit smoothies stored under refrigerated conditions were analysed to determine the bacterial growth during storage. Total counts on day 0 were below the detection limit for the smoothies of exotic fruits, but was very high (4.8 $\log$ CFU mL $\mathrm{m}^{-1}$ ) for the red fruit smoothies. During cold storage (28 days) no growth was detected in the PCA plates from the exotic smoothies samples whereas the counts in the red fruit ones did not change, exceeding the acceptable limit of $3.0 \log \mathrm{CFU} \mathrm{mL} \mathrm{L}^{-1}$, as defined by EU legislation (EU, 2005). These differences between the two types of smoothies may be due to pre-processing of the fruits. In the case of exotic fruit smoothie, mango and passion fruit were peeled before juice extraction while for the red fruit smoothie, one of their ingredients, the raspberry, was processed without any previous treatment. Thus, it would be recommended to include a decontamination step for the raspberries in order to decrease the initial microbial counts.

\section{Sorting task}

In this study we employed the sorting approach in order to check if untrained participants (regular smoothie consumers) could perceive two replicates of the freshly bottle product $(\mathrm{t} 0)$ as similar, and different from samples with longer storage time. The number of smoothie groups formed by the participants varied between two and three, the maximum number of smoothies per group being four. The similarity matrix obtained from the sensory sorting task resulted in the MDS representations shown in Figures 1 and 2.

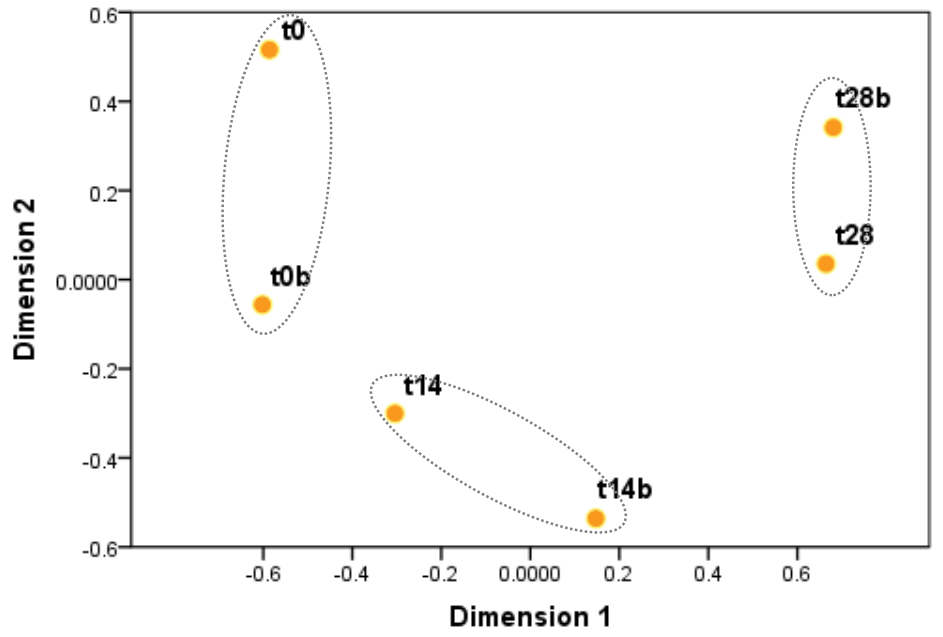

Figure 1. MDS bidimiensional plot of exotic fruit smoothies at t0, t14 and t28 and their replicate sample (indicated as b ("bis")).

\section{Exotic fruits smoothie}

The stress value of the two dimensions MDS representation (Figure 1) was as low as 0.004 . The clustering yielded by HCA clearly shows that replicate samples of t0, t14 and t28 are grouped together following a "time gradient" according to the first dimension (D1). The projection of both $\mathrm{t} 0$, and $\mathrm{t} 28$ replicates, lies onto almost the same D1 coordinate, which means that from a holistic viewpoint, participants perceived the following as conclusive;

i) replicate smoothies corresponding to the initial ( $\mathrm{t} 0$ and $\mathrm{t} 0 \mathrm{~b}$ ) and final ( $\mathrm{t} 28$ and $\mathrm{t} 28 \mathrm{~b}$ ) storage times were considered as extremely similar;

ii) samples freshly bottled presented quite different global sensory characteristics or quality properties from those having been stored for 28 days. 


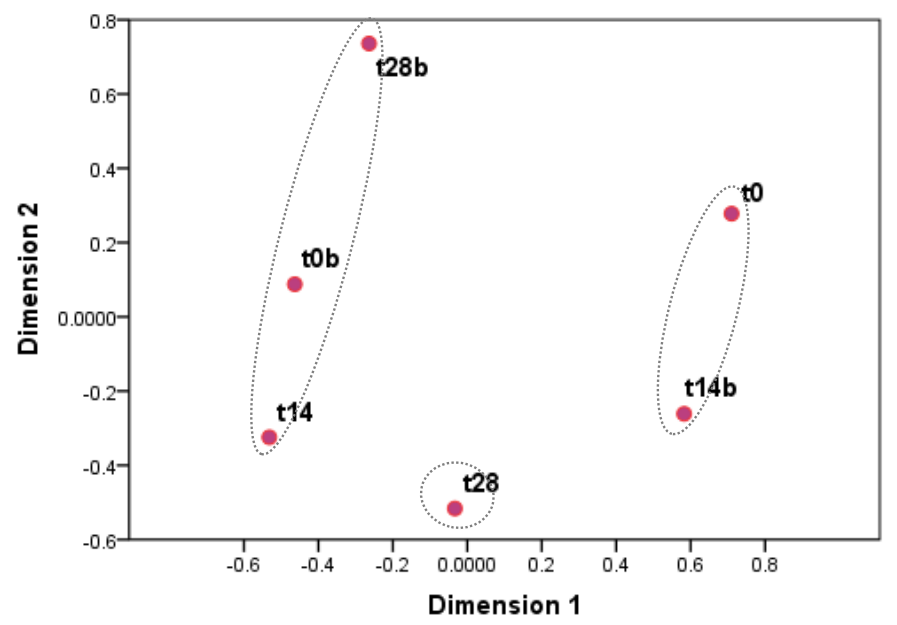

Figure 2. MDS bi-dimensional plot of red fruit smoothies at t0, t14 and t28 and their replicate sample (indicated as b ("bis")).

\section{Red fruits smoothie}

No clustering of smoothies according to the storage time was observed (Figure 2). Taking into account dimension 1 , within the first cluster, a sample of each storage time (t0b, $\mathrm{t} 14$ and $\mathrm{t} 28$ ) appeared clearly differentiated. This means that, contrary to that observed in the exotic fruit flavour (where the first dimension was able to discriminate among smoothies from 0,14 and 28 days), participants were not able to distinguish among the organoleptic properties evoked by these samples, not even between those freshly bottled and those with a higher storage time.

In an attempt to understand the strategy followed by the participants to sort samples we compiled the terms provided to describe the groups. This vocabulary analysis was carried out for the exotic fruit smoothie - and not for the red fruit - as a clustering of samples according to time storage could be deduced from the sorting experiment. Only terms cited by, at least, 8 participants (25\% of the panel) were retained. From those, synonyms were regrouped before data counting (for example thick and dense). Participants qualified some of their descriptors with quantitative terms such as ("very", "slightly", "more" or "less").

No descriptors related to the colour or to the flavour and associated to the specific ingredients (exotic fruits) of this smoothie were employed by participants. Instead, the descriptors belonged to three main sensory aspects according to the total number of quotes received; i) taste (acidity and sweetness); ii) overall flavour intensity/persistence, and iii) texture properties (thickness).

i) Taste. Changes related to the balance between acidity and sweetness were perceived with time storage. Smoothies freshly bottled were globally considered as more balanced, as they had comparable number of quotes for the acid and sweet descriptors. An increase in the storage time yielded to rather unbalanced samples evoking a higher acidity ( 11 quotes, 8 of them with the "very" intensity score, and a very low perception of the sweetness (only 2 quotes). The t14 sample showed a half-way behaviour between fresh and old samples, receiving 9 quotes for the acid, and a total of 6 for the sweet descriptor.

ii) Overall flavour intensity/persistence. Participants generally employed the term intensity with aroma (for example, "intense aroma"), whereas persistence was generally associated to a flavour sensation ("persistence flavour" or "intense aftertaste"). Nevertheless, as they both evoked a degree in which the aromas are perceived either in an ortho, or retronasal way, it was decided to consider them as synonyms and group them in a single term. Smoothies at t0 were those with a higher number of intensity quotes (4 times with the "very" and 2 times with "more" 
intensifying adverbs). No intensity terms were compiled in the t14 samples, and only 3 quotes were given to the $t 28$ samples, this time with the attenuative adverbs "low" or "less". These results show that a gradual decrease of the aroma and flavour intensity is perceived over time.

iii) Thickness. This was the textural descriptor most evoked by participants, but no differences could be observed among samples. This suggests that, even if participants considered the texture important when establishing their groups, no clear pattern could be established with respect to the changes of the smoothie thickness with storage time.

\section{Descriptive analysis}

An important aspect of the present work was to establish attributes characterizing the freshly bottle product (t0), when it is expected to present its optimum properties. For this reason, a trained panel evaluated the smoothies under development, as well as commercial smoothies freshly prepared by our supplier. The projection of the sensory variables on the PCA map is presented in Figure 3.

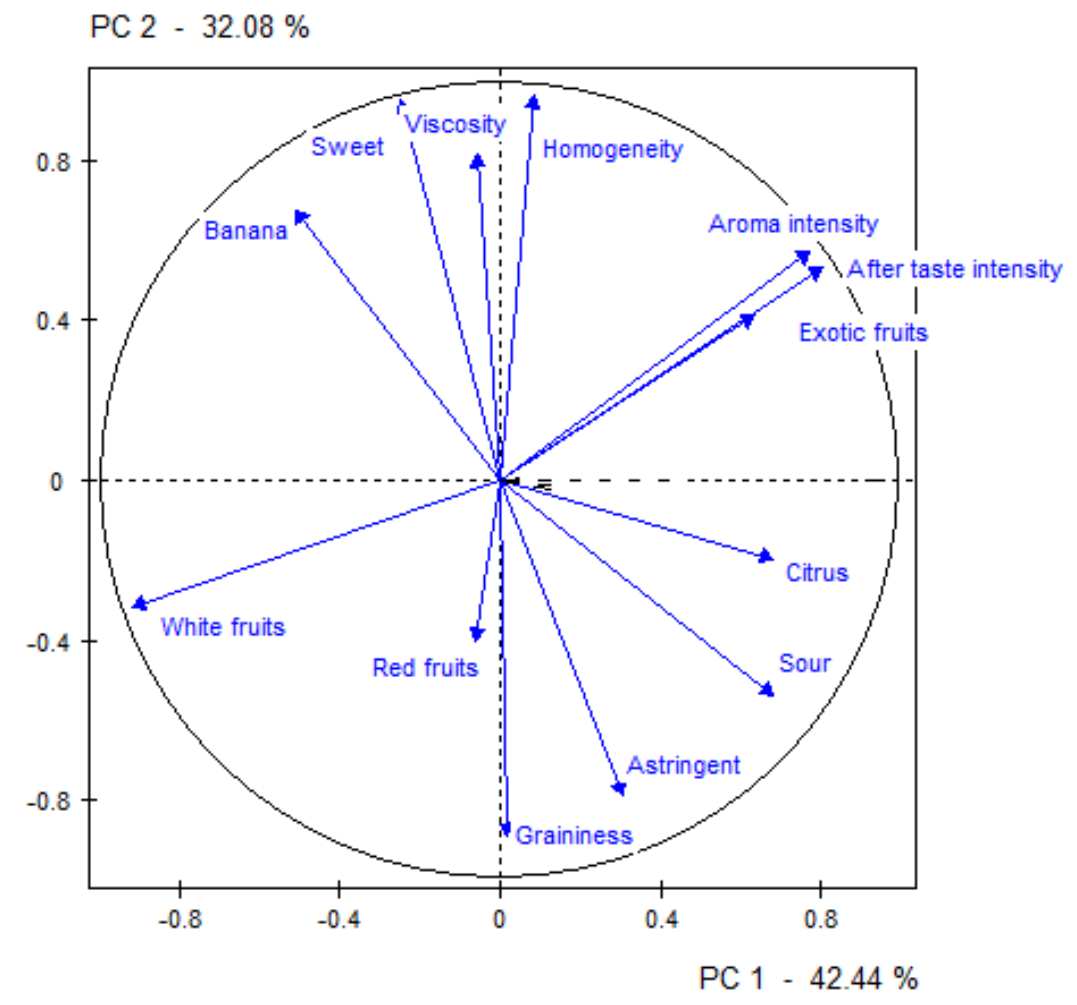

Figure 3. Correlation circle for sensory descriptors in PCA (principal components 1,2).

The first component (43\%) is defined by the overall aroma and after taste intensity, followed by the exotic fruit and citrus aromas, and to a lesser extent, to the sour taste. The second dimension (32\% of explained variance) was mainly defined by textural parameters, opposing the viscosity and homogeneity to the graininess term. Smoothies with a higher viscosity were perceived as the most homogenous.

The projection of samples is presented in Figure 4. The smoothie from red fruits and apple was perceived as the most "liquid-like", with low values of viscosity (1.3/9) and with the higher degree of graininess of all samples evaluated. These results are in accordance with the low content fruit pulp (syneresis parameter), only 14\%. The exotic fruits prototype was projected on the centre area of the map, at a relative distance from the smoothies available in the market with similar ingredients, which suggest that the global flavor intensity of this 
product (still under development) was not as intense as its commercial equivalents.

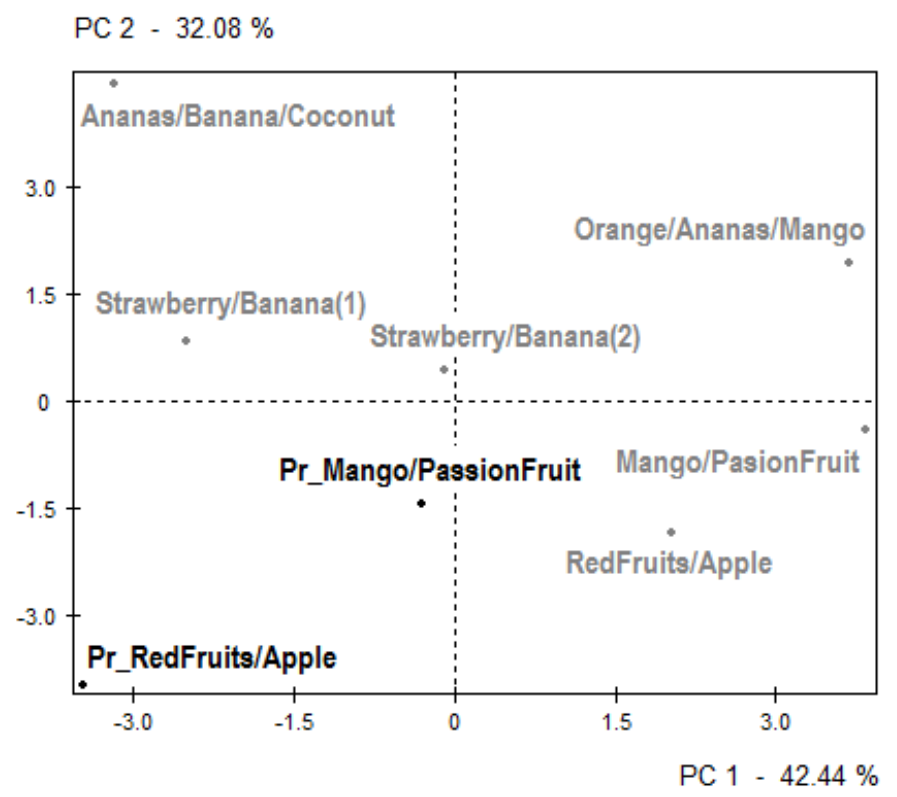

Figure 4. Projection of smoothies in the PCA plot (principal components 1 and 2). Highlighted in black letters "prototype (PR)" smoothies under development.

\section{DISCUSSION}

\section{Exotic fruits smoothie}

The consumers succeeded in sorting the exotic fruit smoothies according to a time gradient, and were able to recognize the replicates of the initial and final storage times as very similar. Panellists pointed out three characteristics that allowed them to discriminate among samples: i) loss of balance between the acidity and the sweetness, ii) decrease of the overall aroma and after-taste intensity, and iii) changes with respect to the density, although no clear pattern with time could be established. All these properties were the same as those underlined by PCA yielded by descriptive analysis as the most relevant to characterize products freshly bottled (Figure 3). The intensity of aroma and after taste conformed to PC1 whereas the textural properties, viscosity - a synonym of the term density provided in the sorting task - and homogeneity vs. graininess defined PC2.

The imbalance between the sweetness and acidity reported by consumers in smoothies after 28 days of storage could be a consequence of the significant decrease of the ${ }^{\circ} \mathrm{Brix}$, and a slight increase of the acidity in this sample, since the importance of both parameters to guarantee a compromise between the sweet and acid perception is well known (Tournier et al., 2007). Taking into account the loss of sensory quality at t28, a maximum of 2 weeks of cold storage should not be exceeded for such sample.

\section{Red fruits smoothie}

Contrary to the exotic fruit flavor, a discrimination of smoothies over time could not be established for this product. The sorting results seem to be in line with both the microbial data and the sensory descriptors from the trained panel, which indicated a poor quality of the freshly bottled product. This smoothie presented low flavour intensity, high graininess and low viscosity (it was the poorest in fruit pulp content), a pool of characteristics that consumers rejected. It can be expected that a product with initial poor quality will not get worse, or to the least, it did not deteriorate enough to be noticed by consumers. 
The different results observed for the smoothies under development may be due to the ingredients employed as raw material. The red fruit smoothie was prepared from strawberries and raspberries, which are added without peeling to the beverage blend. This fact makes the mix more susceptible to microbial growth. This observation is not applied to smoothies only containing peeled fruits (passion fruit, mango or pineapple) as the surface is the main source of microorganisms.

\section{CONCLUSIONS}

In conclusion, without either any pre-established list of terms or training period, consumers were able to define the main attributes responsible for sensory deterioration occurring during storage. This study has shown that when the product complied with an initial quality standard, the sorting procedure is able to monitor product changes over time. This methodology could be adopted by producers that cannot afford maintaining a descriptive panel, which is common for many small food companies.

\section{ACKNOWLEDGEMENTS}

This work was supported by the European Project Life +12 ENV/ES/000902 "ZERO RESIDUES" and the "Department of Industry and Innovation" from the Aragón Government and the European Social Fund. Project 229402/1 (Plant Food Research Group).

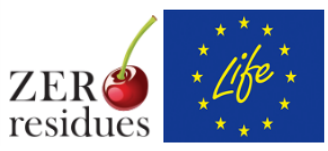

\section{Literature cited}

Chollet, S., Lelievre, M., Abdi, H., and Valentin, D. (2011). Sort and beer: everything you wanted to know about the sorting task but did not dare to ask. Food Qual. Prefer. 22 (6), 507-520 https://doi.org/10.1016/j.foodqual.2011. 02.004 .

EU. (2005). Commission regulation (EC) No 2073/2005 of 15 November 2005 on the microbiological criteria of foodstuffs. Official Journal of the European Union. http://eur-lex.europa.eu/LexUriServ/LexUriServ.do?uri=0J:L: 2005:338:0001:0026:EN:PDF.

Hough, G., and Garitta, L. (2012). Methodology for sensory shelf-life estimation: a review. J. Sens. Stud. 27 (3), 137147 https://doi.org/10.1111/j.1745-459X.2012.00383.x.

ISO 6564:1985. Sensory analysis. Methodology. Flavour profile methods.

ISO 8586-1:1993. Sensory analysis. General guidance for the selection, training and monitoring of assessors. Part 1: Selected assessors.

Macfie, H.J., Bratchell, N., Greenhoff, K., and Vallis, L.V. (1989). Designs to balance the effects of order of presentation and first order carry-over effects in hall test. J. Sens. Stud. 4 (2), 129-148 https://doi.org/10.1111/j.1745459X.1989.tb00463.x.

Tournier, C., Sulmont-Rossé, C., and Guichard, E. (2007). Flavour perception: aroma, taste and texture interactions. A review. Food Global Science Books 1 (2), 246-257.

Varela, P., and Ares, G. (2012). Sensory profiling, the blurred line between sensory and consumer science. A review of novel methods for product characterization. Food Res. Int. 48 (2), 893-908 https://doi.org/10.1016/j.foodres. 2012.06.037. 